\title{
Tangent-Free Property for Periodic Cells Generated by Some General Piecewise Isometries
}

\author{
Rongzhong $\mathrm{Yu} \mathrm{u}^{1}$ and Xinchu $\mathrm{Fu}^{2}$ \\ ${ }^{1}$ College of Sciences, Jiujiang University, Jiujiang 332005, China \\ ${ }^{2}$ Department of Mathematics, Shanghai University, Shanghai 200444, China
}

Correspondence should be addressed to Xinchu Fu; xcfu@shu.edu.cn

Received 20 December 2012; Revised 8 April 2013; Accepted 9 April 2013

Academic Editor: Jonathan Deane

Copyright (C) 2013 R. Yu and X. Fu. This is an open access article distributed under the Creative Commons Attribution License, which permits unrestricted use, distribution, and reproduction in any medium, provided the original work is properly cited.

Iterating an orientation-preserving piecewise isometry $T$ of $n$-dimensional Euclidean space, the phase space can be partitioned with full measure into the union of the rational set consisting of periodically coded points, and the complement of the rational set is usually called the exceptional set. The tangencies between the periodic cells have been studied in some previous papers, and the results showed that almost all disk packings for certain families of planar piecewise isometries have no tangencies. In this paper, the authors further investigate the structure of any periodic cells for a general piecewise isometry of even dimensional Euclidean space and the tangencies between the periodic cells. First, we show that each periodic cell is a symmetrical body to a center if the piecewise isometry is irrational; this result is a generalization of the results in some previously published papers. Second, we show that the periodic cell packing induced by an invertible irrational planar piecewise rotation, such as the Sigma-Delta map and the overflow map, has no tangencies. And furthermore, we generalize the result to general even dimensional Euclidean spaces. Our results generalize and strengthen former research results on this topic.

\section{Introduction}

Piecewise isometries (or PWIs in short) appear in a variety of contexts, including digital filters $[1,2]$, overflow oscillators [3], Hamiltonian systems, and dual billiards. Recently, some researchers investigated this area from a pure mathematics point of view, and PWIs can be treated as the natural generalizations of interval exchange transformations (IETs) discussed in details in [4-7].

In [8-10], some planar PWIs are studied systematically with a particular focus on geometry and symbolic dynamics. In [11-13], the singularity structure and the Devaney-chaos of 2-dimensional invertible PWIs are discussed by classifying singularity into three types with respect to their geometrical properties. In [14], the stability of periodic cells of planar piecewise rotations is investigated; the results showed that the periodic cells are stable if the planar piecewise rotation is irrational. Moreover, in $[15,16]$, the stability of periodic points of a general piecewise isometry of Euclidean space $\mathbb{R}^{n}$ is discussed by using the Euclidean group structure of isometries, and by which we will investigate the structure of periodic cells of general PWIs in this paper. In our opinion, the Euclidean group of isometries is an important and helpful method to study the dynamics of general PWIs of Euclidean space $\mathbb{R}^{d}$.

Iterating an orientation-preserving piecewise isometry $T$ of $n$-dimensional Euclidean space $\mathbb{R}^{n}$, the phase space can be partitioned with full measure into the union of the rational set consisting of all periodically coded points, and its complement is called the exceptional set. For a planar irrational piecewise isometry (the rotation parameter $\theta$ is incommensurable with $\pi$ ), a periodic cell of all points with the same periodic coding is a disk $[14,17,18]$; hence, the rational set gives rise to a disk packing of the phase space $M$. A natural problem is whether any two periodic cells are tangent (see Figure 1).

For the tangent-free property of disk packing induced by planar PWIs, some results have been presented in previous papers. All these previous results have suggested that the packing is typically very "loose"; that is, there are almost no tangencies between any two disks, and this supports 


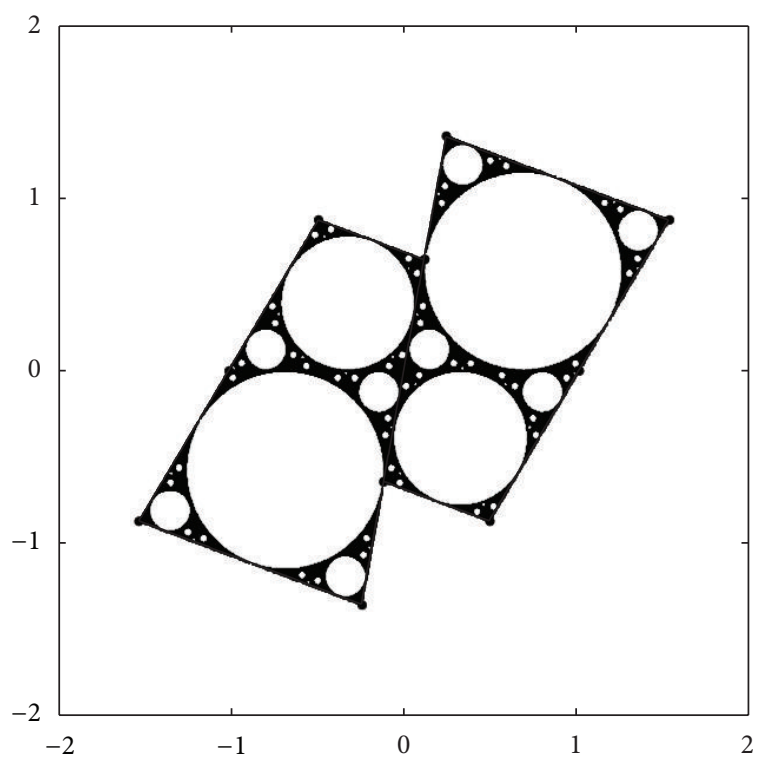

(a)

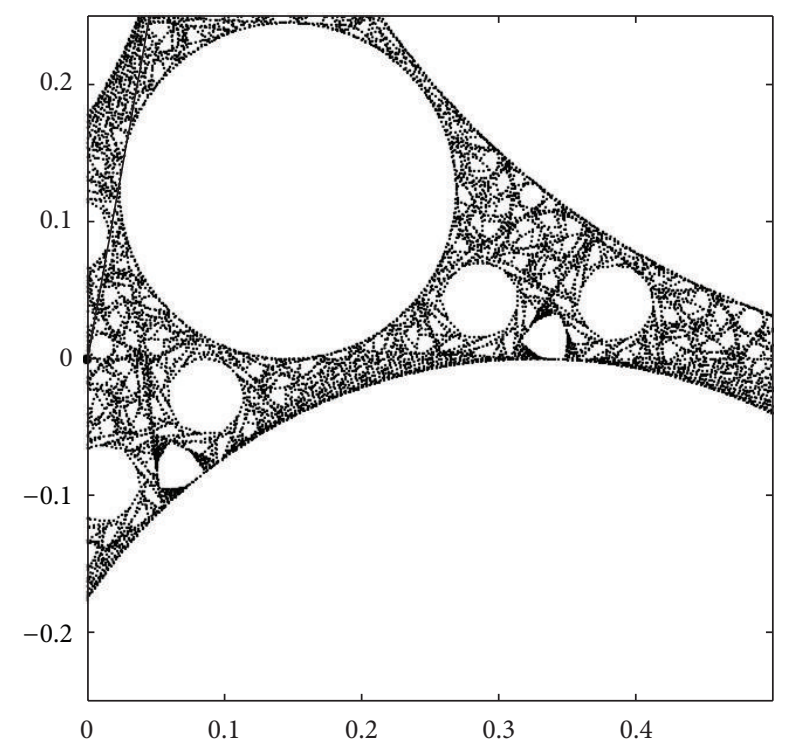

(b)

FIGURE 1: (a) The illustration to the invariant disks packing of the Sigma-Delta map for the parameter $\theta=1.75$. (b) The local magnification of the square $[0,0.5] \times[-0.25,0.25]$, we can see that the disk packing is very "loose" and there is no tangencies between any pair of invariant disks.

the conjecture that the irrational set has positive measure for an irrational planar piecewise isometry. In [18] it is shown that the disk packings induced by invariant periodic cells cannot contain certain Apollonian packings, namely, the Arbelos. It is revealed in [19] that for planar irrational piecewise rotations, only finitely many tangencies are possible to any disk. In $[17,20]$, the tangent-free property of disk packing induced by a one-parameter family of PWIs (the Sigma-Delta map and the Overflow map) is investigated, and the results (as summarized in Theorem A below) showed that tangencies between disks in this packing are rare, and this was proven by discussing analytic functions of the parameters.

Theorem A (see, Fu et al's [17, 20]). There is at most a countable set of $\theta$ for which there are any tangencies between periodic disk in the disk packing induced by the overflow map (the Sigma-Delta map). Therefore, there is a full measure set of $\theta$ such that the disk packing induced by the Overflow map (the Sigma-Delta map) has no tangencies.

In [21], it is shown that the result above can be generalized to some quite general planar PWIs under some reasonable constraints (Theorem B below).

Theorem B (see, Fu et al.s [21]). There is a countable set of $\theta$ in the parameter range for which there are no tangencies between the invariant periodic disks in the disk packing induced by a PWI; therefore, there is a full measure of $\theta$ such that the disk packing has no tangencies.

Although the previous results show that all tangencies may occur at a countable set of parameter values, the possibility that a dense set of parameters do have tangencies is not excluded. Naturally, we have the following questions.

(Q1) Whether does the disk packing induced by a planar PWI has no tangencies if the rotation parameter $\theta$ is incommensurable with $\pi$ ?

(Q2) How to characterize the tangent-free property between periodic cells for higher dimensional PWIs?

In this paper, we will focus on the above questions. For Question one (Q1), we will give a positive answer which is one special issue of higher dimensional PWIs if $n$ is even. For Question two (Q2), we must at first investigate the structure of the periodic cells induced by a general higher dimensional piecewise isometry. As mentioned in some previous papers $[14,17,18]$, a periodic cell of planar PWIs is either a disk (the rotation parameter $\theta$ is incommensurable with $\pi$ ) or a polygonal region (the rotation parameter $\theta$ is commensurable with $\pi$ ). However, the structure of a periodic cell of a higher dimensional PWI is more complicated. In Section 3.5, we show that a periodic cell may be represented as the product space of two disks. For generality, we show in Theorem 3 that the closure of any periodic cell is symmetrical about the center (the unique fix point) if $n$ is even and the piecewise isometry is irrational. Moreover, we obtain the main result (Theorem 8, Section 3.2) about the tangent-free property of the periodic cell packing induced by higher dimensional PWIs.

In particular, we confirm that for any invertible planar irrational piecewise rotations, such as the Sigma-Delta map and the Overflow map, there are no tangencies between periodic disks in the disk packing. Our results are more general and more precise than some previous known results presented in $[17,20,21]$ (e.g., Theorem A and Theorem B), and our proofs are simpler.

The rest of this paper is organized as follows. In Section 2, we will introduce the preliminaries about piecewise isometries, including the definitions of PWIs, codings, and cells. 
In Section 3, we investigate the periodic cells and their tangent-free properties, which are the main part of this paper. And some examples are also provided in this section, and it can be found that it is very easy to verify that these example systems have no tangencies according to our results. Furthermore, a 4-dimensional PWI is investigated in Section 3.5, and it is shown that any periodic cell is the product space of two disks, and there exist no tangencies between two periodic cells. Finally, in Section 4, we present some remarks and discussions about our research in this paper.

\section{Preliminaries}

The structure of the Euclidean groups plays an important role for the discussion of high-dimensional piecewise isometries. We briefly recall some notations about piecewise isometries and the structure of the Euclidean groups as papers $[15,22]$ in this part.

The Euclidean group $\mathbb{E}(n)=\mathbb{O}(n) \ltimes \mathbb{R}^{n}$ is the semidirect product of the orthogonal group $\mathbb{O}(n)$ and $\mathbb{R}^{n}$, where $\mathbb{O}(n)=$ $\left\{A \in \mathbb{G} \mathbb{L}(n, \mathbb{R}): A A^{t}=I d\right\}$. Denote an element of $g \in \mathbb{E}(n)$ as a pair $(A, v)$ where $A \in \mathbb{O}(n)$ and $v \in \mathbb{R}^{n}$. The group multiplication is given by $\left(A_{1}, v_{1}\right) \cdot\left(A_{2}, v_{2}\right)=$ $\left(A_{1} A_{2}, A_{1} v_{2}+v_{1}\right)$. The subgroup $\mathbb{S O}(n) \subset \mathbb{O}(n)$ called special orthogonal group consists of orientation-preserving transformations, defined by $\mathbb{S O O}(n)=\{A \in \mathbb{O}(n) \mid \operatorname{det}(A)=$ $1\}$. The special Euclidean group $\mathbb{S} \mathbb{E}(n)$ is the semidirect product of $\mathbb{S O}(n)$ and $\mathbb{R}^{n}$.

Let $M$ be a connected subset of Euclidean space $\mathbb{R}^{n}$ and let $\mathscr{M}=\left\{M_{0}, M_{1}, \ldots, M_{r-1}\right\}$ be a finite collection of connected open convex set. We call $\mathscr{M}$ a partition of $M$ and each set $M_{i}$ a partition atom if the following conditions hold:

(1) $M=\bar{M}_{1} \cup \cdots \cup \bar{M}_{r-1}$;

(2) $M_{i} \cap M_{j}=\emptyset$ for $i \neq j$.

A map $T: M \backslash \mathscr{D} \mapsto M$ is called a piecewise isometry on $M$ if $T(x)=T_{i}(x), x \in M_{i}$, where $T_{i} \in \mathbb{E}(n)$. And $\mathscr{D}=\cup_{i \neq j}\left(\bar{M}_{i} \cap \bar{M}_{j}\right)$ is said to be the discontinuity set. For convenience, we denote by $\mathbb{E}(\mathscr{M})$ the set of all piecewise isometries and by $\mathbb{S} \mathbb{E}(\mathscr{M})$ the set of all orientation-preserving piecewise isometries on the partition $\mathscr{M}$.

In this paper, we consider the case where the orientationpreserving piecewise isometries $\left(T_{i} \in \mathbb{S} \mathbb{E}(n), i=0,1, \ldots, r-\right.$ $1)$ and each of the partition atoms $M_{i}(i=0,1, \ldots, r-1)$ is an open convex polyhedron of $\mathbb{R}^{n}$ which is surrounded by $m_{i}$ number of $(n-1)$-dimensional hyperplanes taking the form $\mathbf{n}_{i j} \cdot x=c_{i j}\left(j=1,2, \ldots, m_{i}\right)$, where $\mathbf{n}_{i j}$ representing the unit normal vector of the hyperplane is an element of the projective space $\mathbb{P}^{n-1}$ (i.e., ignoring the difference between $\pm \mathbf{n}), x=\left(x_{1}, x_{2}, \ldots, x_{n}\right)^{T} \in \mathbb{R}^{n}$ is a column vector, and $c_{i j} \in \mathbb{R}$ is a constant. We say that two unit vectors $\mathbf{n}$ and $\mathbf{n}^{\prime}$ are equal means that $\mathbf{n}=\mathbf{n}^{\prime}$ or $\mathbf{n}=-\mathbf{n}^{\prime}$. In [18], the authors characterized similarly the boundary of partition atoms for planar PWIs.

Let $\partial \mathscr{M}=\cup_{i=0}^{r} \partial M_{i}$ be the boundary of the partition $\mathscr{M}$ and $\mathscr{D}^{\prime}=\cup_{i \neq j}\left(\overline{T\left(M_{i}\right)} \cap \overline{T\left(M_{j}\right)}\right)$ the backward discontinuity set. A piecewise isometry is said to be invertible if
$T\left(M_{i}\right) \cap T\left(M_{j}\right)=\emptyset(i \neq j)$ and $\cup_{i=0}^{r-1} \overline{T\left(M_{i}\right)}=M$. Obviously, all of the sets $\partial M, \mathscr{D}$, and $\mathscr{D}^{\prime}$ consist of a finite number of $(n-1)$-dimensional polyhedral regions for invertible PWIs.

In the following, for convenience, we say $L$ is a $(n-1)$ dimensional hyperplane of $\partial \mathscr{M}$ (or $\mathscr{D}, \mathscr{D}^{\prime}$ ), if $L \subset \partial \mathscr{M}$ (or $\left.\mathscr{D}, \mathscr{D}^{\prime}\right)$ is a $(n-1)$-dimensional polyhedral region of $\partial \mathscr{M}$ (or $\mathscr{D}, \mathscr{D}^{\prime}$ ). For a $(n-1)$-dimensional hyperplane $L$, let $\mathbf{n}(L)$ be a unit normal vector of $L$ and $\mathbf{N}(\mathscr{M})=: \cup_{L \subset \partial \mathscr{M}}\{\mathbf{n}(L)\}=$ $\cup_{i=0}^{r-1} \cup_{j=1}^{m_{i}}\left\{\mathbf{n}_{i j}\right\}$ the set of all unit normal vectors of hyperplanes of $\mathscr{M}$. Similarly, we define $\mathbf{N}(\mathscr{D})$ and $\mathbf{N}\left(\mathscr{D}^{\prime}\right)$.

The partition $\mathscr{M}=\left\{M_{0}, M_{1}, \ldots, M_{r-1}\right\}$ of $M$ associated with a piecewise isometry $T$ gives rise to a natural one-sided coding map. Let $\mathscr{E}^{-}=\cup_{k=0}^{\infty} T^{-k}(\mathscr{D})$ and $\mathscr{A}=\{0,1, \ldots, r-1\}$ the alphabet set; a map $\chi: M \backslash \mathscr{E}^{-} \mapsto \mathscr{A}^{\mathbb{N}}$ is called a coding map, if for any point $x \in M \backslash \mathscr{E}^{-}$,

$$
[\chi(x)]_{j}=\omega_{j}, \quad \text { iff } T^{j}(x) \in M_{\omega_{j}}, j=0,1,2, \ldots
$$

And we call the infinite sequence $\omega=\omega_{0} \omega_{1} \omega_{2} \ldots$ the coding of the orbit $\left\{T^{j}(x)\right\}_{j=0}^{\infty}$ (or the coding of the point $x$ for simplicity). An infinite sequence $\omega=\omega_{0} \omega_{1} \ldots$ is said to be admissible, if there exists a point $x$ such that $\chi(x)=\alpha$. Similarly, a finite sequence $\beta=\beta_{0} \beta_{1} \cdots \beta_{n-1}$ with length $n$ is said to be admissible, if there exists a point $x$ such that $\beta$ is the preword of $\chi(x)$. A coding $\omega$ is said to be periodic, if there exists a natural number $p$ such that $\omega_{p+k}=\omega_{k}$ for all $k \in \mathbb{N}$, and we denote the coding $\omega$ by $\omega=\mathscr{P}\left(\omega_{0} \omega_{1} \cdots \omega_{p-1}\right)$, where $\mathscr{P}(\cdot)$ represents concatenation operation. A coding $\omega$ is said to be rational, if it is eventually periodic. Otherwise, the coding $\omega$ is said to be irrational. In fact, for an invertible piecewise isometry, a rational coding is periodic. Sometimes, we may denote $j$-dimensional coding as paper [23] for convenience. Namely, let the alphabet set $\mathscr{A}=$ $\left\{\alpha_{0}, \alpha_{1}, \ldots, \alpha_{r-1}\right\}$ and every element $\alpha_{i}=\left(\alpha_{i 1}, \alpha_{i 2}, \ldots, \alpha_{i j}\right)$ is a $j$-dimensional vector.

Definition 1. If a coding $\omega$ (finite or infinite) is admissible, we call the set of all points following the same coding $\omega$ a cell, denoted by $C(\omega)$.

The structure of cells of planar PWIs has been investigated by some researchers; some of the results are stated in the following proposition.

Proposition 2 (see $[9,14,17,18,24])$. For a planar piecewise isometry with convex polygonal partition, a cell $C(\omega)$ is a convex set. More precisely, it has interior if and only if $\omega$ is rational, and if $\omega$ is irrational, then the cell is either a point or a line segment.

In [25], it is shown that for an irrational piecewise rotation, if the admissible coding $\omega$ is irrational, then the cell $C(\omega)$ consists of only a point.

Let $T_{\omega}=T_{\omega_{m-1}} \cdot T_{\omega_{m-2}} \cdots T_{\omega_{0}}$ and $A_{\omega}=A_{\omega_{m-1}} \cdot A_{\omega_{m-2}} \cdots$ $A_{\omega_{0}}$ for every finite coding. A piecewise isometry $T \in \mathbb{S} \mathbb{E}(\mathscr{M})$ is said to be incommensurate if Fix $A_{\omega}=\{0\}$ for every finite word $\omega$ in the alphabet $\mathscr{A}$. As mentioned in [15], if $n$ is even then almost every piecewise isometry in $\mathbb{S E}(\mathscr{M})$ is incommensurate. 


\section{Periodic Cells and the Tangent-Free Property}

Based on the fact revealed by Mendes and Nicol in [15] that if $n$ is odd, then almost every piecewise isometry in $\mathbb{S} \mathbb{E}(\mathscr{M})$ has no recurrent points with rational coding (consequently, has no periodic points), and we will only discuss the periodic cells and the tangent-free property of the even dimensional piecewise isometries in this part.

3.1. Periodic Cells. If $n$ is even and $\omega=\omega_{0} \cdots \omega_{m-1}$ is admissible, then the linear part $A_{\omega}$ of the map $T_{\omega}=\left(A_{\omega}, v\right)$ can be orthogonally diagonalized as $\operatorname{diag}\left\{B_{1}, B_{2}, \ldots, B_{n / 2}\right\}$ under one orthogonal basis, where $B_{j}=\left(\cos \theta_{j}, \sin \theta_{j} ;-\sin \theta_{j}, \cos \theta_{j}\right)$, $\theta_{j} \in[0,2 \pi)$. It is obvious that an even dimensional orientation-preserving PWI is incommensurate if and only if all $\theta_{j}$ are incommensurable with $\pi$ in diagonalization representation of $A_{\omega}$ for every finite word $\omega$. Furthermore, we say the PWI is irrational if it is incommensurable and all $\theta_{j}$ are rationally independent in diagonalization representation of $A_{\omega}$ for every finite word $\omega$.

Let $\omega=\mathscr{P}(\alpha)$ be an admissible periodic coding, where $\alpha=$ $\omega_{0} \omega_{1} \cdots \omega_{m-1}$ is of length $m$, then the self-map $T_{\alpha}: C(\omega) \mapsto$ $C(\omega)$ can be represented as the product of $n / 2$ number of planar rotations since the linear part $A_{\alpha}$ can be orthogonally diagonalized. Namely, every point of $C(\omega)$ can be rewritten as complex number coordinate $z=\left(z_{1}, z_{2}, \ldots, z_{n / 2}\right)$, and the map $T_{\alpha}$ can be represented as $T_{\alpha}=\widetilde{T}_{1} \times \widetilde{T}_{2} \times \cdots \times \widetilde{T}_{n / 2}$, $\widetilde{T}_{j}\left(z_{j}\right)=e^{i \cdot \theta_{j}}+c_{j}, j=1,2, \ldots, n / 2$. We have the following result.

Theorem 3. Suppose $n$ is even, $T \in \mathbb{S E}(\mathscr{M})$, then every periodic cell is convex and of positive Lebesgue measure. Furthermore, if $T$ is irrational then the closure of the periodic cell is centrosymmetric with respect to a unique fixed point.

Proof. The proof of the convexity of the cell is similar to the one given in [18]; here we almost repeat the procedure. Let $\omega=\mathscr{P}(\alpha)$ be an admissible periodic coding, where $\alpha=\omega_{0} \omega_{1} \cdots \omega_{m-1}$ is of length $m$, then the cell $C(\omega)$ can be represented as

$$
\begin{aligned}
C(\omega) & =\{x: \chi(x)=\omega\} \\
& =\left\{x: x \in M_{\omega_{0}}, T^{k}(x) \in M_{\omega_{k}} \text { for } k \in \mathbb{N}\right\} \\
& =M_{\omega_{0}} \cap T^{-1}\left(M_{\omega_{1}}\right) \cap T^{-2}\left(M_{\omega_{1}}\right) \cap \cdots \\
& =\bigcap_{k=0}^{\infty} T^{-k}\left(M_{\omega_{k}}\right) .
\end{aligned}
$$

At the same time, we have

$$
T^{-k}\left(M_{\omega_{k}}\right)=T_{\omega_{1}}^{-1} \circ \cdots \circ T_{\omega_{k}}^{-1}\left(M_{\omega_{k}}\right),
$$

where $M_{\omega_{k}}$ is convex and each of the maps $\left\{T_{\omega_{1}}^{-1}, T_{\omega_{2}}^{-1}, \ldots, T_{\omega_{k}}^{-1}\right\}$ is an isometry, then the set $T^{-k}\left(M_{\omega_{k}}\right)$ is convex, consequently, the cell $C(\omega)$ is convex.
Since the map $T_{\alpha}: C(\omega) \mapsto C(\omega)$ is isometric and $T_{\alpha}(C(\omega))=C(\omega)$, the cell $C(\omega)$ at least contains a fixed point denoted by $p^{*}$. Let

$$
d=\frac{1}{2} \min _{0 \leq i \leq p-1}\left\{\rho\left(T^{i}\left(p^{*}\right), \partial \mathscr{M}\right)\right\},
$$

then $B\left(p^{*}, d\right) \subset C(\omega)$, which implies that the cell $C(\omega)$ is of positive Lebesgue measure.

Now, we prove the latter claim. Suppose, without loss of generality, the map $T_{\alpha}$ is represented as the product of $n / 2$ planar rotations and every point of $C(\omega)$ has the form of complex coordinate. Furthermore, assume the unique fixed point equals $\mathbf{0}$ of the map $T_{\alpha}$ since the piecewise isometry $T$ is irrational. We will show that $z=\left\{z_{1}, z_{2}, \ldots, z_{n / 2}\right\} \in \overline{C(\omega)}$ implies that every point $z^{\prime}=\left\{z_{1}^{\prime}, z_{2}^{\prime}, \ldots, z_{n / 2}^{\prime}\right\} \in \overline{C(\omega)}$ with $\left\|z_{i}^{\prime}\right\|=\left\|z_{i}\right\|\left(1 \leq i \leq r_{2}\right)$, (i.e., $\left.\mathbf{T}^{n / 2} \subset \overline{C(\omega)}\right)$, where

$$
\begin{aligned}
\mathbf{T}^{n / 2}= & \left\{\left(\left\|z_{1}\right\| e^{\alpha_{1}},\left\|z_{2}\right\| e^{\alpha_{2}}, \ldots,\left\|z_{r_{2}}\right\| e^{\alpha_{n / 2}}\right) \mid \alpha_{i} \in[0,2 \pi),\right. \\
& \left.i=1,2, \ldots, r_{2}\right\}
\end{aligned}
$$

is the $(n / 2)$-dimensional torus in the phase space. For $z=$ $\left\{z_{1}, z_{2}, \ldots, z_{n / 2}\right\} \in \overline{C(\omega)}$, and there exists a point $z^{\star}=\left\{z_{1}^{\star}\right.$, $\left.z_{2}^{\star}, \ldots, z_{n / 2}^{\star}\right\} \in C(\omega)$ such that for any $\epsilon>0$,

$$
\left\|z-z^{\star}\right\|<\frac{\epsilon}{n}
$$

Let

$$
\begin{gathered}
\mathbf{T}_{1}^{n / 2}=\left\{\left(\left\|z_{1}^{\star}\right\| e^{\alpha_{1}},\left\|z_{2}^{\star}\right\| e^{\alpha_{2}}, \ldots,\left\|z_{r_{2}}^{\star}\right\| e^{\alpha_{n / 2}}\right) \mid \alpha_{i} \in[0,2 \pi),\right. \\
\left.i=1,2, \ldots, \frac{n}{2}\right\}
\end{gathered}
$$

be another torus in the phase space. At the same time, the orbit $\operatorname{Orb}^{+}\left(z^{\star}\right)$ under the map $T_{\alpha}: C(\omega) \rightarrow C(\omega)$ is dense in the torus $\mathbf{T}_{1}^{n / 2}$ because the map $T$ is irrational. For any point $z^{\prime}=\left(z_{1}^{\prime}, \ldots, z_{n / 2}^{\prime}\right) \in \mathbf{T}^{n / 2}$, then $z^{\prime \prime}=\left(\left\|z_{1}^{\star} / z_{1}^{\prime}\right\| z_{1}^{\prime}, \ldots\right.$, $\left.\left\|z_{i}^{\star} / z_{i}^{\prime}\right\| z_{i}^{\prime}, \ldots,\left\|z_{n / 2}^{\star} / z_{n / 2}^{\prime}\right\| z_{n / 2}^{\prime}\right) \in \mathbf{T}_{1}^{n / 2}$. Consequently, there exists a natural number $k$ such that

$$
\left\|T_{\alpha}^{k}\left(z^{\star}\right)-z\right\|<\frac{\epsilon}{2}
$$

Because

$$
\begin{aligned}
\left\|z_{i}^{\prime}-\right\| \frac{z_{i}^{\star}}{z_{i}^{\prime}}\left\|\cdot z_{i}^{\prime}\right\| & =\left\|\frac{\left\|z_{i}^{\prime}\right\|-\left\|z_{i}^{\star}\right\|}{\left\|z_{i}^{\prime}\right\|} \cdot z_{i}^{\prime}\right\| \\
& =\left|\left\|z_{i}^{\prime}\right\|-\left\|z_{i}^{\star}\right\|\right| \\
& =\left|\left\|z_{i}\right\|-\left\|z_{i}^{\star}\right\|\right| \\
& \leq\left\|z_{i}-z_{i}^{\star}\right\|
\end{aligned}
$$


for all $i=1,2, \ldots, n / 2$, from (9) and (6) we obtain

$$
\left\|z^{\prime}-z\right\| \leq \sum_{i=1}^{n / 2}\left\|z_{i}^{\prime}-\right\| \frac{z_{i}^{\star}}{z_{i}^{\prime}}\left\|\cdot z_{i}^{\prime}\right\| \leq \sum_{i=1}^{n / 2}\left\|z_{i}-z_{i}^{\star}\right\| \leq \frac{\epsilon}{2} .
$$

Consequently, from (8) and (10), we have

$$
\left\|T_{\alpha}^{k}\left(z^{\star}\right)-z^{\prime}\right\| \leq\left\|T_{\alpha}^{k}\left(z^{\star}\right)-z\right\|+\left\|z^{\prime}-z\right\|<\frac{\epsilon}{2}+\frac{\epsilon}{2}=\epsilon,
$$

namely, $z^{\prime} \in \overline{C(\omega)}$. Consequently, the periodic cell is centrosymmetric with respect to the unique fixed point.

Remark 4. According to Theorem 3, since the set $\overline{C(\omega)}$ is convex, then $z=\left(z_{1}, z_{2}, \ldots, z_{n / 2}\right) \in \overline{C(\omega)}$ implies $z^{\prime}=$ $\left(z_{1}^{\prime}, z_{2}^{\prime}, \ldots, z_{n / 2}^{\prime}\right) \in \overline{C(\omega)}$ with $\left\|z_{i}^{\prime}\right\| \leq\left\|z_{i}\right\|$ for all $1 \leq i \leq n / 2$. Let $\Theta(z)=\left\{z^{\prime} \mid\left\|z_{i}^{\prime}\right\| \leq\left\|z_{i}\right\|, i=1, \ldots, n / 2\right\}$ be the product of $n / 2$ number of discs, then the closure of the periodic cell $C(\omega)$ is the union of some such sets as $\Theta(z)$.

Let $\gamma_{i}=\max \left\{\left\|z_{i}\right\| \mid\left(z_{1}, \ldots, z_{i}, \ldots, z_{n / 2}\right) \in \overline{C(\omega)}\right\}$ for all $i=1,2, \ldots, n / 2$, and $\Theta=\left\{z=\left(z_{1}, z_{2}, \ldots, z_{n / 2}\right) \mid\left\|z_{i}\right\| \leq\right.$ $\left.\gamma_{i}, i=1,2, \ldots, n / 2\right\}$. According to the analysis above, we can obtain that $\overline{C(\omega)}=\Theta$, which is the product of $n / 2$ discs. We have the following corollary.

Corollary 5. Suppose $n$ is even, $T \in \mathbb{S} \mathbb{E}(\mathscr{M})$ is irrational, if there exists a point $z=\left(z_{1}, z_{2}, \ldots, z_{n / 2}\right)$ with $\left\|z_{i}\right\|=\left\|\gamma_{i}\right\|(i=$ $1, \ldots, n / 2)$ such that $z \in \overline{C(\omega)}$, then $\overline{C(\omega)}=\Theta$ is the product of $n / 2$ number of discs.

Note that the result in the above theorem is a generalization of the results in $[14,17,18]$, where it is shown that a periodic cell is a disk or a symmetric polygonal region. Obviously, for a 3-dimensional piecewise isometry with polygonal partition, a periodic cell must not be a sphere. In Section 3.5, we will show that a periodic cell of the product Goetz map, which is a 4-dimensional PWI, may be represented as $C(\omega) \times$ $C(\omega)$, where $\omega, \omega$ are two admissible periodic codings under the Goetz map. For higher-dimensional PWIs, the structure of a periodic cell is more complicated. In the following, we further investigate the periodic cells.

At the same time, for higher-dimensional PWIs, their periodic cells have some similar properties as the ones of planar PWIs.

Proposition 6. For a piecewise isometry $T \in \mathbb{S} \mathbb{E}(\mathscr{M})$, if $C(\omega)$ and $C(\omega)$ are two different periodic cells, then there must exist a natural number $k$ such that $T^{k}(C(\omega))$ and $T^{k}(C(\omega))$ are contained in different partition atoms $M_{i}$ and $M_{j}$, respectively; that is, they will be separated by an $(n-1)$-dimensional boundary $L \subset \mathscr{D}$.

We must point out that two periodic cells are separated by an $(n-1)$-dimensional boundary $L \subset \mathscr{D}$ as above means that $\left(\mathbf{n} \cdot x^{\star}-c\right) \cdot\left(\mathbf{n} \cdot x^{\star \star}-c\right)<0$, where $x^{\star}$ and $x^{\star \star}$ are the centers of two periodic cells, respectively, and $\mathbf{n} \cdot x=c$ is the equation of hyperplane $L$. Note that Proposition 6 is a generalization of
Lemma 1 in [17], and the proof is similar. More importantly, for an invertible piecewise isometry, we have another similar property stated as follows.

Proposition 7. For a piecewise isometry $T \in \mathbb{S} \mathbb{E}(\mathscr{M})$, if $C(\omega)$ and $C(\omega)$ are two different periodic cells, then there must exist a natural number $k$ such that $T^{-k}(C(\omega))$ and $T^{-k}(C(\omega))$ are separated by an $(n-1)$-dimensional polyhedral region $L^{\prime} \subset \mathscr{D}^{\prime}$.

Proof. Assume that the periodic codings $\omega$ and $\omega$ have periods of $N_{0}$ and $N_{1}$, respectively, and further assume, without loss of generality, that $N_{1} \geq N_{0}$. Consider the preimages of the pair of periodic cells $C(\omega)$ and $C(\omega)$, if $T^{-k-1}(C(\omega))$ and $T^{-k-1}(C(\omega))$ locate in different atoms, then $T^{-k}(C(\omega))$ and $T^{-k}(C(\omega))$ are separated by a hyperplane $L^{\prime} \subset \mathscr{D}^{\prime}$. In fact, it can be verified that such $k$ (less than $\left[N_{0}, N_{1}\right]$, the least common multiple of $N_{0}$ and $N_{1}$ ) exists since $\omega \neq \omega$.

3.2. Tangencies between Periodic Cells. Similar to the planar cases $[17,20,21]$, for a $n$-dimensional PWI ( $n \geq 4$ is even), we say that the set $X=\bigcup C(\omega)$, the union of all periodic cells, is a periodic cell packing of the phase space $M$ by the $d$ dimensional PWI. We consider similarly the tangent property between any two periodic cells. Assume that the boundaries of the periodic cells $C_{1}$ and $C_{2}$ are smooth at the intersection point $x$, and we say the two periodic cells $C_{1}$ and $C_{2}$ are tangent to each other at the point $x$ if

(1) $\operatorname{int}\left(C_{1}\right) \cap \operatorname{int}\left(C_{2}\right)=\emptyset$;

(2) $x \in \partial C_{1} \cap \partial C_{2}$;

(3) $\mathbf{n}_{1}(x)= \pm \mathbf{n}_{2}(x)$, where $\mathbf{n}_{i}(x)(i=1,2)$ represent the unit normal vectors of surface $\partial C_{i}$ at the point $x$.

The above three conditions are obvious; the fact that any two different periodic cells do not overlap implies Condition (1); and Conditions (2) and (3) guarantee they are tangent at $x$. Let

$$
\mathscr{T}\left(C_{1}, C_{2}\right)=\left\{x \mid x \text { is atangent point of } C_{1} \text { and } C_{2}\right\}
$$

be the mutually tangent set. We say the periodic cells $C_{1}$ and $C_{2}$ are tangent, if $\mathscr{T}\left(C_{1}, C_{2}\right)$ is nonempty. We say the packing is tangent-free, if any two periodic cells are not tangent.

In fact, the mutually tangent set $\mathscr{T}\left(C_{1}, C_{2}\right)$ may be a single point or an open straight line segment for a planar piecewise isometry, and it may be a $k$-dimensional $(k=$ $0,1, \ldots, n-1)$ hyperplane for a general piecewise isometry of $n$-dimensional Euclidean space $\mathbb{R}^{n}$. In the following, we give another main result, which will be proven later in Section 3.2 after preparing some propositions.

Theorem 8. Suppose $n$ is even, $T \in \mathbb{S} \mathbb{E}(\mathscr{M})$ is invertible and incommensurate. If for any admissible finite coding $\omega=$ $\omega_{0} \omega_{1} \cdots \omega_{k}$ and any $\mathbf{n}, \mathbf{n}^{\prime}$ of $\mathbf{N}\left(\mathscr{D}^{\prime}\right)$, one has $A_{\omega} \cdot \mathbf{n} \neq \mathbf{n}^{\prime}$, then there are no tangencies between periodic cells in the periodic cells packing induced by $T$. 
For a class of PWIs, the backward discontinuity set consists of one hyperplane or finitely many parallel hyperplanes. Namely, $\mathbf{N}\left(\mathscr{D}^{\prime}\right)$ consists of only one element without considering the sign. Then we get the following corollary.

Corollary 9. Suppose $n$ is even, $T \in \mathbb{S} \mathbb{E}(\mathscr{M})$ is invertible and incommensurate. If $\mathbf{N}\left(\mathscr{D}^{\prime}\right)$ consists of only one element, then there are no tangencies between periodic cells in the periodic cells packing induced by $T$.

For convenience of expression, we give some notations introduced in [19]. Two admissible $n$-periodic codings $\omega$ and $\omega$ are said to be equivalent, denoted by $\omega \sim \omega$, if there exists a natural number $i(0 \leq i \leq n)$ such that $\sigma^{i}(\omega)=\omega$, where $\sigma$ is the shift map. Two points of $M$ are said to be equivalent, if their corresponding symbolic sequences are equivalent. Similarly, we can define the equivalence of two cells $C(\omega)$ and $C(\omega)$. We denote by $\operatorname{GO}(C(\omega))$ the set of all the cells which are equivalent to $C(\omega)$ under the above equivalence relation and call $\mathrm{GO}(C(\omega))$ the great orbit of $C(\omega)$. According to Propositions 6 and 7, we have the following result.

Proposition 10. Suppose $n$ is even, $T \in \mathbb{S} \mathbb{E}(\mathscr{M})$ is invertible, if two different periodic cells $C(\omega)$ and $C(\omega)$ are tangent to each other, then there exist two hyperplanes $L_{1} \subset \mathscr{D}^{\prime}, L_{2} \subset \mathscr{D}$ and periodic cells $C_{1}, C_{2} \in \mathrm{GO}(C(\omega)), C_{1}^{\prime}, C_{2}^{\prime} \in \mathrm{GO}(C(\omega))$ such that $C_{i}$ and $C_{i}^{\prime}(i=1,2)$ are tangent to each other, and the mutually tangent set $\mathscr{T}\left(C_{i}, C_{i}^{\prime}\right)$ is contained in $L_{i}$ for $i=1,2$, respectively.

From the above result, we further have the following.

Proposition 11. Suppose $n$ is even, $T \in \mathbb{S} \mathbb{E}(\mathscr{M})$ is invertible, if two different periodic cells $C(\omega)$ and $C(\omega)$ are tangent to each other, then there exist two vectors $\mathbf{n}_{1}$ and $\mathbf{n}_{2}$ of $\mathbf{N}(\mathscr{M})$ and an admissible coding $\alpha=\alpha_{0} \cdots \alpha_{k-1}$ with length $k$ such that $A_{\alpha}$. $\mathbf{n}_{1}=\mathbf{n}_{2}$.

Proof. Without loss of generality, we assume that the mutually tangent set is contained in the hyperplane $L^{\prime} \subset \mathscr{D}^{\prime}$ (i.e., $\left.\mathscr{T}(C(\omega), C(\omega)) \subset L^{\prime}\right)$ with unit normal vector $\mathbf{n}_{1}^{\prime}$ according to Proposition 10 . Then, there is a hyperplane $L_{1} \subset \partial \mathscr{M}$ with unit normal vector $\mathbf{n}_{1}$ such that $A_{\alpha_{0}} \cdot \mathbf{n}_{1}=\mathbf{n}_{1}^{\prime}$. From Proposition 6 , there exists a natural number $k$ such that the periodic cells $T^{k-1}(C(\omega))$ and $T^{k-1}(C(\omega))$ are in the different partition atoms $M_{i}$ and $M_{j}$, respectively, and $T^{k-1}(\mathscr{T}(C(\omega), C(\omega))) \subset$ $L \subset \mathscr{D}$, where $L=\bar{M}_{i} \cap \bar{M}_{j}$. Let $\mathbf{n}_{2}$ be the unit normal vector of the hyperplane $L$, then there exists a finite admissible coding $\alpha_{1} \alpha_{2} \cdots \alpha_{k-1}$ such that $A_{\alpha_{k-1}} \cdots A_{\alpha_{1}} \cdot \mathbf{n}_{1}^{\prime}=\mathbf{n}_{2}$. Consequently,

$$
A_{\alpha} \cdot \mathbf{n}_{1}=A_{\alpha_{k-1}} \cdots A_{\alpha_{1}} A_{\alpha_{0}} \cdot \mathbf{n}_{1}=\mathbf{n}_{2} .
$$

The proof is therefore complete.

We are now ready to give the proof of Theorem 8 .

Proof of Theorem 8. Firstly, we will reveal that there exists a natural number $k_{0}$ such that for any $\mathbf{n}$ of $\mathbf{N}(\mathscr{M})$, there exists an admissible finite coding $\alpha=\alpha_{0} \cdots \alpha_{k-1}$ with length $k \leq k_{0}$ such that $A_{\alpha} \cdot \mathbf{n} \in \mathbf{N}\left(\mathscr{D}^{\prime}\right)$. Since every partition atom is surrounded by finite $(n-1)$-dimensional hyperplanes, then $\mathbf{N}(\mathscr{M})$ consists of finite elements, and let $k_{0}=\sharp \mathbf{N}(\mathscr{M})$ be the cardinal number. Because the isometry is invertible, for any $(n-1)$-dimensional hyperplanes $L \subset \partial \mathscr{M}$, we can get $T(L) \subset \partial \mathscr{M} \cup \mathscr{D}^{\prime}$. If there exists an admissible finite coding $\alpha=\alpha_{0} \cdots \alpha_{k-1}$ with length $k>k_{0}$ such that $T^{i}(L) \subset \partial \mathscr{M}$ for all $i=0, \ldots, k-1$, then $A(i) \cdot \mathbf{n} \in \mathbf{N}(\mathscr{M})$ for all $i=0, \ldots, k-1$, where $A(i)=A_{\alpha_{0}} \cdots A_{\alpha_{i-1}}$. Since the piecewise isometry is incommensurate, all $A(i) \cdot \mathbf{n}$ are different from each other. This implies that $\sharp \mathbf{N}(\mathscr{M}) \geq k>k_{0}$, a contradiction.

Suppose that there exist two periodic cells $C_{1}$ and $C_{2}$ which are tangent to each other, then, in view of Proposition 11, there is two unit normal vectors $\mathbf{n}_{1}$ of a hyperplane $L^{\prime} \subset \mathscr{D}^{\prime}$ and $\mathbf{n}$ of a hyperplane $L \subset \mathscr{D}$, and an admissible finite coding $\beta=\beta_{0} \beta_{1} \cdots \beta_{k-1}$, such that

$$
A_{\beta} \cdot \mathbf{n}_{1}=\mathbf{n}
$$

Via the typical invertibility condition, we can obtain that there exists an admissible finite coding $\alpha=\alpha_{0} \alpha_{1} \cdots \alpha_{l-1}$ and a unit normal vector $\mathbf{n}_{2} \in \mathbf{N}\left(\mathscr{D}^{\prime}\right)$ such that

$$
A_{\alpha} \cdot \mathbf{n}=\mathbf{n}_{2}
$$

According to (14) and (15), we have

$$
A_{\beta} \cdot A_{\alpha} \cdot \mathbf{n}_{1}=\mathbf{n}_{2}
$$

Let $\omega=\alpha \beta=\alpha_{0} \cdots \alpha_{l-1} \beta_{0} \cdots \beta_{k-1}$ with length $k+l$, then we get $A_{\omega} \cdot \mathbf{n}_{1}=\mathbf{n}_{2}$ for two elements of $\mathbf{N}\left(\mathscr{D}^{\prime}\right)$, which contradicts the results as above. Therefore, there are no tangencies between periodic cells.

3.3. Applications to Planar PWIs. For planar piecewise rotations, the corresponding results are very explicit. Let $\rho_{j}=$ $e^{i \cdot \theta_{j}}(j=0,1, \ldots, r-1)$ be the linear part of the map $\left.T\right|_{M_{j}}$, then we have the following corollary.

Corollary 12. Suppose $\mathscr{M} \subset \mathbb{R}^{2}, T \in \mathbb{S} \mathbb{E}(\mathscr{M})$ is invertible, if $\rho_{0}^{k_{0}} \rho_{1}^{k_{1}} \cdots \rho_{r-1}^{k_{r-1}}=1$ with $k_{i} \geq 0(0 \leq i \leq r-1)$ implies that $k_{i}=0$, and if $\mathbf{N}\left(\mathscr{D}^{\prime}\right)$ consists of only one element, then the invariant disk packing is tangent-free.

We note that the above corollary is just a simple case of Corollary 9.

In particular, if an invertible planar piecewise rotation has the common irrational rotation angle $\theta$, that is, any restricted map $\left.T\right|_{M_{j}}$ has the same linear part $\rho=e^{i \cdot \theta}$, then we have the following corollary, which strengthens the results in $[17,20,21]$.

Corollary 13. If an invertible planar piecewise rotation has the common irrational rotation angle $\theta\left(\theta / \pi \in \mathbb{Q}^{C}\right)$ and $\mathbf{N}\left(\mathscr{D}^{\prime}\right)$ consists of only one element, then the invariant disk packing induced by the map is tangent-free. 


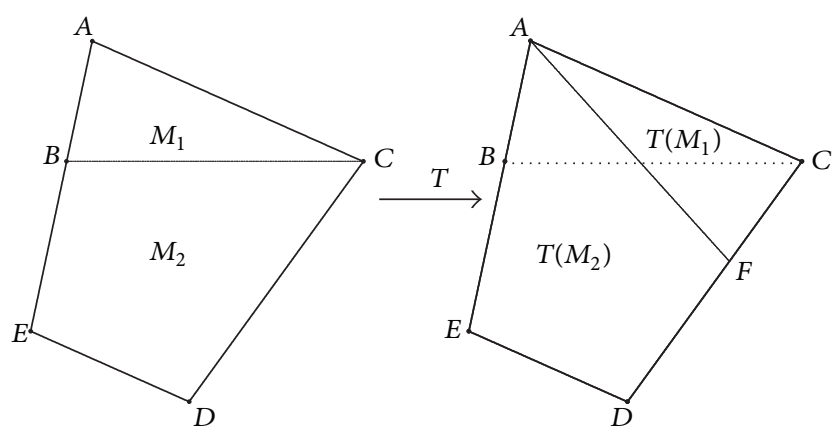

FIgURE 2: The piecewise isometric map (17) has two partition atoms $M_{1}$ and $M_{2}$, and the linear parts of the restricted maps are $\rho_{1}=$ $e^{2(\pi-\theta) i}$ and $\rho_{2}=e^{(\pi-\theta) i}$. At the same time, the map is invertible, that is, $T\left(M_{1}\right) \cup T\left(M_{2}\right)=M$.

3.4. Goetz Map. Now we consider a map called the (bounded) Goetz map $T_{\theta}: M \mapsto M$ with parameter $\theta \in(\pi / 2,2 \pi / 3)$ as follows:

$$
T_{\theta}(z)= \begin{cases}e^{2(\pi-\theta) i} \cdot z+c_{1} & \text { if } z \in M_{1} \\ e^{(\pi-\theta) i} \cdot z+c_{2} & \text { if } z \in M_{2}\end{cases}
$$

Here, $c_{1}=1 / \sin \theta, c_{2}=((1+2 \cos \theta) / \sin \theta) e^{-\theta i}$, and the partition atoms $M_{1}$ and $M_{2}$ can be written as follows (see Figure 2):

$$
\begin{aligned}
& M_{1}=[A, B, C]=\left[-2 \cot \theta \cdot e^{(\pi-\theta) i}, 0, \frac{1}{\sin \theta}\right], \\
M_{2}= & {[B, C, D, E] } \\
= & {\left[0, \frac{1}{\sin \theta}, \frac{1}{\sin \theta} \cdot\left(1+e^{-3 \theta} i\right), \frac{1+2 \cos \theta}{\sin \theta} \cdot e^{-\theta i}\right] . }
\end{aligned}
$$

The piecewise isometric map is invertible, and the backward discontinuity set $\mathscr{D}^{\prime}$ consists of only the line segment $\overline{A F}$; furthermore, all of the boundary line segments of the partition atoms $M_{1}$ and $M_{2}$ will turn on to the line segment $\overline{A F}$ under finite iterations of the map $T$; that is, the isometric map $T$ satisfies the typical invertibility condition. At the same time, the linear parts of the restricted maps are $\rho_{1}=e^{2(\pi-\theta) i}$ and $\rho_{2}=e^{(\pi-\theta) i}$, and obviously, when $\theta / \pi$ is irrational, the equality

$$
\rho_{1}^{k_{1}} \rho_{2}^{k_{2}}=1, \quad k_{1} \geq 0, k_{2} \geq 0
$$

holds if and only if $k_{1}=k_{2}=0$. Then, from Corollary 12, we have the following.

Proposition 14. For the piecewise isometry defined by (17), if the parameter $\theta$ is incommensurable with $\pi$, then the invariant disk packing is tangent-free. (See Figure 3).

3.5. The Product Goetz Map. Now we investigate the socalled product Goetz map $F_{\theta}=T_{\theta} \times T_{\theta}: M \times M \mapsto$ $M \times M$ which is a 4 -dimensional PWI with partition

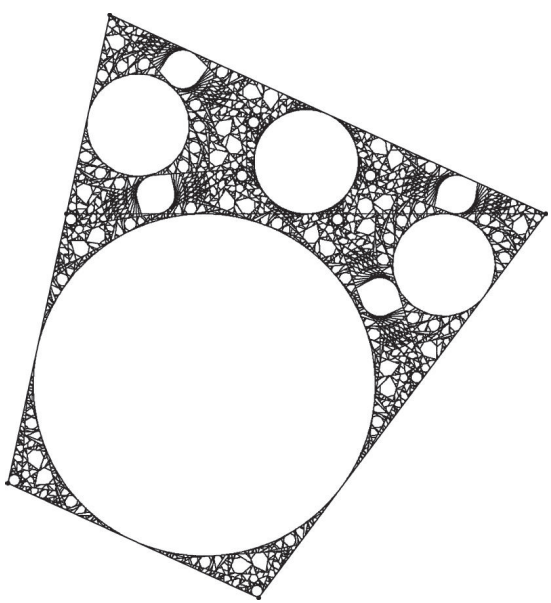

FIgURE 3: The illustration to invariant disk packing induced by the map (17), where the parameter $\theta=1.78$. We can observe that all the periodic disks are tangent-free.

$\mathscr{M} \times \mathscr{M}=\left\{M_{i} \times M_{j}, i=1,2\right\}$. The partition atom $M_{i} \times M_{j}$ is surrounded by $n_{i}+n_{j} 3$-dimensional hyperplanes in $\mathbb{C}^{2}$, where $n_{i}\left(n_{j}\right)$ represents the number of hyperplanes which surround $M_{i}\left(M_{j}\right)$. The boundary of partition atom $M_{i} \times M_{j}$ may be represented as $\left(\partial M_{i} \times M_{j}\right) \cup\left(M_{i} \times \partial M_{j}\right)$, so a point $z=\left(z_{1}, z_{2}\right) \in \partial\left(M_{i} \times M_{j}\right)$ if and only if $z_{1} \in \partial M_{i}, z_{2} \in \bar{M}_{j}$ or $z_{1} \in \bar{M}_{i}, z_{2} \in \partial M_{j}$.

Let $\chi: M \rightarrow M$ be the coding map of the Goetz map taking the form

$$
[\chi(z)]_{i}=\omega_{i} \in\{1,2\} \quad \text { iff } T_{\theta}^{i}(z) \in M_{\omega_{i}} \text { for } i=0,1, \ldots
$$

As mentioned in Section 2, we denote the coding of any point $z$ of the phase space $M \times M$ by $\iota(z)$ with $[\iota(z)]_{i}=\kappa_{i}=\left(\omega_{i}, \omega_{i}\right)$ if $F_{\theta}^{i}(z) \in M_{\omega_{i}} \times M_{\omega_{i}}$, where $\omega_{i}, \omega_{i}=1,2$ for all $i \in \mathbb{N} \cup\{0\}$.

Proposition 15. Let $\Omega$ be the set of all admissible codings of the Goetz map $T_{\theta}$, then a 2-dimensional coding $\kappa$ is admissible under the product Goetz map if and only if $\kappa=\omega \times \omega$, where $\omega, \omega \in \Omega$ and $[\omega \times \omega]_{i}=\left(\omega_{i}, \omega_{i}\right)$. Moreover, if the rotation parameter $\theta$ is incommensurable with $\pi$ and an admissible coding $\kappa=\omega \times \omega$ is periodic, then $p(\kappa)=[p(\omega), p(\omega)]$, the least common multiple of $p(\omega)$ and $p(\omega)$, and the periodic cell $C(\kappa)$ of the product Goetz map may be represented as $C(\kappa)=$ $C(\omega) \times C(\omega)$, where $p(\cdot)$ represents the period of a periodic coding.

Proof. Obviously, if a 2-dimensional coding $\kappa=\left(\omega_{0}\right.$, $\left.\omega_{0}\right)\left(\omega_{1}, \omega_{1}\right) \ldots$ is admissible, then there exists $z=\left(z_{1}, z_{2}\right)$ such that $\iota(z)=\kappa$. Consequently, $T_{\theta}^{i}\left(z_{1}\right) \in M_{\omega_{i}}$ and $T_{\theta}^{i}\left(z_{2}\right) \in$ $M_{\varrho_{i}}$ for all $i=0,1, \ldots$. This implies that the codings $\omega$, $\omega$ are admissible under the map $T_{\theta}$, that is, $\omega, \omega \in \Omega$. At the same time, let $\omega, \omega \in \Omega$ be two admissible codings, then there exist two points $z_{1}, z_{2}$ such that $\chi\left(z_{1}\right)=\omega$ and $\chi\left(z_{2}\right)=\omega$. Let $z=\left(z_{1}, z_{2}\right)$ be a point of the partition atom $M_{\omega_{0}} \times M_{\omega_{0}}$, then $F_{\theta}^{i}(z)=\left(T_{\theta}^{i}\left(z_{1}\right), T_{\theta}^{i}\left(z_{2}\right)\right) \in M_{\omega_{i}} \times M_{\omega_{i}}$; that is, $\kappa=\omega \times \omega$ is admissible under the map $F_{\theta}$. 
It is obvious that $p(\kappa)=[p(\omega), p(\varpi)]$. Now we check $C(\kappa)=C(\omega) \times C(\omega)$. For convenience, we only consider the case for $\omega=\Phi=1^{\infty}$ (the other cases can be verified similarly). Let $z^{\star}$ be the center, and $r$ the radius of the periodic disk $C(\omega)(C(\omega))$. On one hand, for every point $z=\left(z_{1}, z_{2}\right)$ with $\left\|z_{i}-z^{\star}\right\|<r, i=1,2$, we have $T_{\theta}^{n}\left(z_{i}\right) \in M_{1}$ for $i=1,2, n \in \mathbb{N} \cup\{0\}$ since $z_{i} \in C(\omega)=C(\omega)$, then $F_{\theta}^{n}(z) \in C(\kappa)$ for all $n \in \mathbb{N} \cup\{0\}$. Thus, $z \in C(\omega) \times C(\varpi)$. On the other hand, for every point $z=\left(z_{1}, z_{2}\right)$ with $\left\|z_{1}-z^{\star}\right\|>r$ or $\left\|z_{2}-z^{\star}\right\|>r$, we will show that $z \notin C(\kappa)$. Without loss of generality, we suppose that $\left\|\mathrm{z}_{1}-z^{\star}\right\|>r$, then there exists a natural number $n_{0}$ such that $T_{\theta}^{n_{0}}\left(z_{1}\right) \notin M_{1}$; this implies that $F_{\theta}^{n_{0}}(z)=\left\{T_{\theta}^{n_{0}}\left(z_{1}\right), T_{\theta}^{n_{0}}\left(z_{2}\right)\right\} \notin C(\kappa)$.

In fact, we can find that $D_{1} \subset C(\kappa) \subset D_{2}$ for $\kappa=(1,1)^{\infty}$, where

$$
\begin{gathered}
D_{1}=\left\{\left(z_{1}, z_{2}\right) \mid\left\|z_{1}-z^{\star}\right\|+\left\|z_{1}-z^{\star}\right\|<r\right\}, \\
D_{2}=\left\{\left(z_{1}, z_{2}\right) \mid\left\|z_{1}-z^{\star}\right\|+\left\|z_{1}-z^{\star}\right\|<\frac{\sqrt{2}}{2} r\right\} .
\end{gathered}
$$

More precisely, the boundary $\partial C(\kappa)$ of the periodic cell $C(\kappa)$ consists of $C(\omega) \times \partial C(\omega)$ and $\partial C(\omega) \times C(\omega)$; however, the 2-dimensional torus $\partial C(\omega) \times \partial C(\Phi)$ is merely a fraction of $\partial C(\kappa)$.

We consider below the tangent-free property between any two periodic cells. Obviously, for any 3-dimensional boundary $L \subset \partial(\mathscr{M} \times \mathscr{M})$, the unit normal vector may be represented as $\mathbf{n}(L)=(-\sin \alpha, \cos \alpha, 0,0)$ or $\mathbf{n}(L)=$ $(0,0,-\sin \alpha, \cos \alpha)$, where $\tan \alpha$ is a slope of a line segment of $\partial \mathscr{M}$. Moreover, the backward discontinuity set $\mathscr{D}^{\prime}$ consists of two 3-dimensional polyhedral regions $L_{1}=\overline{A F} \times M$ and $L_{2}=M \times \overline{A F}$, with $\mathbf{n}\left(L_{1}\right)=(-\sin \beta, \cos \beta, 0,0)$, $\mathbf{n}\left(L_{2}\right)=(0,0,-\sin \beta, \cos \beta)$, where $\tan \beta$ is the slope of the line segment $\overline{A F}$. By the analysis in Section 3.4, we can obtain that there exists a natural number $k_{0}$ such that for every point $z \in \partial(\mathscr{M} \times \mathscr{M}), F_{\theta}^{k}(z) \in L_{1} \cup L_{2}$ for some $k \leq k_{0}$. Let

$$
A_{(i, j)}=\left(\begin{array}{cccc}
\cos \alpha_{i} & -\sin \alpha_{i} & 0 & 0 \\
\sin \alpha_{i} & \cos \alpha_{i} & 0 & 0 \\
0 & 0 & \cos \alpha_{j} & -\sin \alpha_{j} \\
0 & 0 & \sin \alpha_{j} & \cos \alpha_{j}
\end{array}\right)
$$

for $i, j=1,2$, where $\alpha_{1}=2 \pi-\theta, \alpha_{2}=\pi-\theta$, then $A_{(i, j)}$ is the linear part of the restricted map $\left.F_{\theta}\right|_{M_{i} \times M_{j}}$. If the rotation parameter $\theta$ is incommensurable with $\pi$, then for any admissible coding $\kappa=\left(\omega_{0}, \oplus_{0}\right)\left(\omega_{1}, \oplus_{1}\right) \ldots$ we have $A_{\kappa} \cdot \mathbf{n}\left(L_{i}\right) \neq \mathbf{n}\left(L_{j}\right)$ for all $k \in \mathbb{N}$ and $i, j=1,2$. Therefore, from Theorem 8 , we have the following proposition.

Proposition 16. If $\theta$ is incommensurable with $\pi$, then the periodic cell packing induced by the product Goetz map $F_{\theta}$ is tangent-free.

\section{Discussions}

As we know, for planar irrational piecewise rotations, a periodic cell is a disk except from possible countable points on the boundary, while for higher-dimensional PWIs, we do not think that a periodic cell is a sphere. In fact, it is easy to see that, for odd dimensional PWIs with polygonal partition, a periodic cell is not a sphere. By the same way as the product Goetz map, we have the following results.

Proposition 17. Let $T \in \mathbb{S} \mathbb{E}(\mathscr{M})$ and $F \in \mathbb{S} \mathbb{E}(\mathscr{X})$ be two PWIs and $H=T \times F: M \times X \rightarrow M \times X$, every periodic cell $C(\kappa)$ of $H$ can be written as $C(\omega) \times C(\varpi)$, where $\omega$ and $\omega$ are two admissible periodic codings of $T$ and $F$, respectively.

Proposition 18. $\operatorname{Let} T^{i} \in \mathbb{S} \mathbb{E}\left(\mathscr{M}^{i}\right)(i=1,2, \ldots, k)$ be k planar PWIs and $H=T^{1} \times T^{2} \times \cdots \times T^{k}$, if each of them is an irrational rotation, then every periodic cell $C$ of the map $H$ can be represented as $C=C_{1} \times C_{2} \times \cdots \times C_{k}$, where $C_{i}$ is a periodic disk of the map $T^{i}$ for all $i=1,2, \ldots, k$. Moreover, the periodic cell packing induced by the PWI H is tangent-free.

Generally, for a PWI of even-dimensional Euclidean space, we guess that every periodic cell may be written as the topological product space of $n / 2$ disks under a continuous translation. To confirm this, further research is needed.

As mentioned in Section 1, we can confirm here that the disk packing induced by the Sigma-Delta map $[20,26]$ and the overflow map [17] are tangent-free for all the parameters $\theta$ which are incommensurable with $\pi$. In fact, it is now easy to check that the two piecewise isometries satisfy the conditions of Corollary 13. So we just state the results as follows.

Proposition 19. For the Sigma-Delta map and the Overflow map with $\theta / \pi$ irrational, the induced disk packings are tangentfree.

\section{Acknowledgments}

This research was jointly supported by NSFC Grant 11072136 and Shanghai University Leading Academic Discipline Project (A.13-0101-12-004), and a grant of "The First-class Discipline of Universities in Shanghai." Rongzhong Yu was also supported by a science and technology Project of Jiangxi Province, Department of Education (GJJ12617 and GJJ13714). The authors would also like to thank the anonymous referees for their critical but very helpful comments and suggestions.

\section{References}

[1] L. O. Chua and T. Lin, "Chaos in digital filters," IEEE Transactions on Circuits and Systems, vol. 35, no. 6, pp. 648-658, 1988.

[2] L. Kocarev, C. W. Wu, and L. O. Chua, "Complex behavior in digital filters with overflow nonlinearity: analytical results," IEEE Transactions on Circuits and Systems II, vol. 43, no. 3, pp. 234-246, 1996.

[3] P. Ashwin, "Non-smooth invariant circles in digital overflow oscillations," in Proceedings of the 4th International Workshop on Nonlinear Dynamics of Electronic Systems, pp. 417-422, Sevilla, Spain, 1996.

[4] H. Haller, "Rectangle exchange transformations," Monatshefte für Mathematik, vol. 91, no. 3, pp. 215-232, 1981. 
[5] M. Keane, "Interval exchange transformations," Mathematische Zeitschrift, vol. 141, pp. 25-31, 1975.

[6] W. A. Veech, "Interval exchange transformations," Journal d’Analyse Mathématique, vol. 33, pp. 222-272, 1978.

[7] W. A. Veech, "Gauss measures for transformations on the space of interval exchange maps," Annals of Mathematics. Second Series, vol. 115, no. 1, pp. 201-242, 1982.

[8] A. Goetz, "Dynamics of a piecewise rotation," Discrete and Continuous Dynamical Systems, vol. 4, no. 4, pp. 593-608, 1998.

[9] A. Goetz, "Dynamics of piecewise isometries," Illinois Journal of Mathematics, vol. 44, no. 3, pp. 465-478, 2000.

[10] P. Ashwin and X.-C. Fu, "On the geometry of orientationpreserving planar piecewise isometries," Journal of Nonlinear Science, vol. 12, no. 3, pp. 207-240, 2002.

[11] B. Kahng, "Redefining chaos: devaney-chaos for piecewise continuous dynamical systems," International Journal of Mathematical Models and Methods in Applied Sciences, vol. 3, no. 4, pp. 317-326, 2009.

[12] B. Kanhng, "On Denvaney's definiton of chaos for discontinuous dynamical systems," Advances in Applied Mathematics, vol. 15, pp. 89-94, 2009.

[13] B. Kahng, "Singularities of two-dimensional invertible piecewise isometric dynamics," Chaos, vol. 19, no. 2, article 023115, 2009.

[14] A. Goetz, "Stability of piecewise rotations and affine maps," Nonlinearity, vol. 14, no. 2, pp. 205-219, 2001.

[15] M. Mendes and M. Nicol, "Periodicity and recurrence in piecewise rotations of Euclidean spaces," International Journal of Bifurcation and Chaos in Applied Sciences and Engineering, vol. 14, no. 7, pp. 2353-2361, 2004.

[16] M. S. Mendes, "Quasi-invariant attractors of piecewise isometric systems," Discrete and Continuous Dynamical Systems A, vol. 9, no. 2, pp. 323-338, 2003.

[17] P. Ashwin and X.-C. Fu, "Tangencies in invariant disc packings for certain planar piecewise isometries are rare," Dynamical Systems, vol. 16, no. 4, pp. 333-345, 2001.

[18] M. Trovati, P. Ashwin, and N. Byott, "Packings induced by piecewise isometries cannot contain the Arbelos," Discrete and Continuous Dynamical Systems A, vol. 22, no. 3, pp. 791-806, 2008.

[19] R.-Z. Yu, X.-C. Fu, and S.-L. Shui, "Density of invariant disk packings for planar piecewise isometries," Dynamical Systems, vol. 22, no. 1, pp. 65-72, 2007.

[20] P. Ashwin, X.-C. Fu, and J. Deane, "Properties of the invariant disk packing in a model bandpass sigma-delta modulator," International Journal of Bifurcation and Chaos in Applied Sciences and Engineering, vol. 13, no. 3, pp. 631-641, 2003.

[21] X.-C. Fu, S.-L. Shui, and L.-G. Yuan, “Tangents-free property in invariant disk packings generated by some general planar piecewise isometries," Chaos, Solitons and Fractals, vol. 36, no. 1, pp. 115-120, 2008.

[22] M. S. Mendes, "Stability of periodic points in piecewise isometries of Euclidean spaces," Ergodic Theory and Dynamical Systems, vol. 27, no. 1, pp. 183-197, 2007.

[23] X.-C. Fu and P. Ashwin, "Symbolic analysis for some planar piecewise linear maps," Discrete and Continuous Dynamical Systems A, vol. 9, no. 6, pp. 1533-1548, 2003.

[24] L. Kocarev, C. W. Wu, and L. O. Chua, "Complex behavior in digital filters with overflow nonlinearity: analytical results," IEEE Transactions on Circuits and Systems II, vol. 43, no. 3, pp. 234-246, 1996.
[25] R.-Z. Yu, X.-C. Fu, and L. Ye, "On codings and dynamics of planar piecewise rotations," Physica D, vol. 240, no. 21, pp. 17851790, 2011.

[26] P. Ashwin, J. H. B. Deane, and X. C. Fu, "Dynamics of a bandpass sigma-delta modulator as a piecewise isometry," in Proceedings of the IEEE International Symposium on Circuits and Systems (ISCAS '01), pp. 811-814, Sydney, Australia, May 2001. 


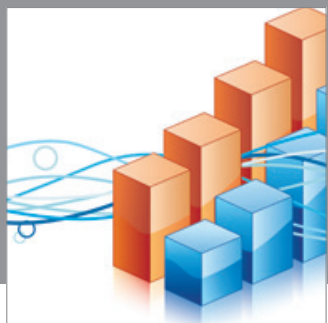

Advances in

Operations Research

mansans

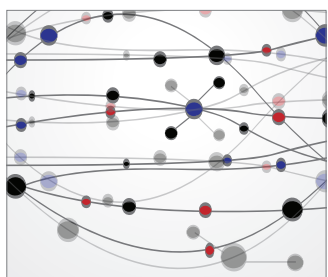

The Scientific World Journal
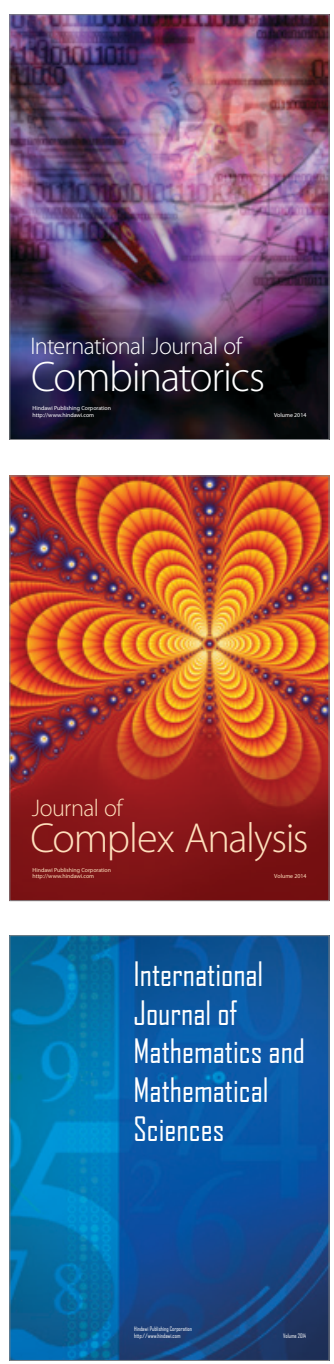
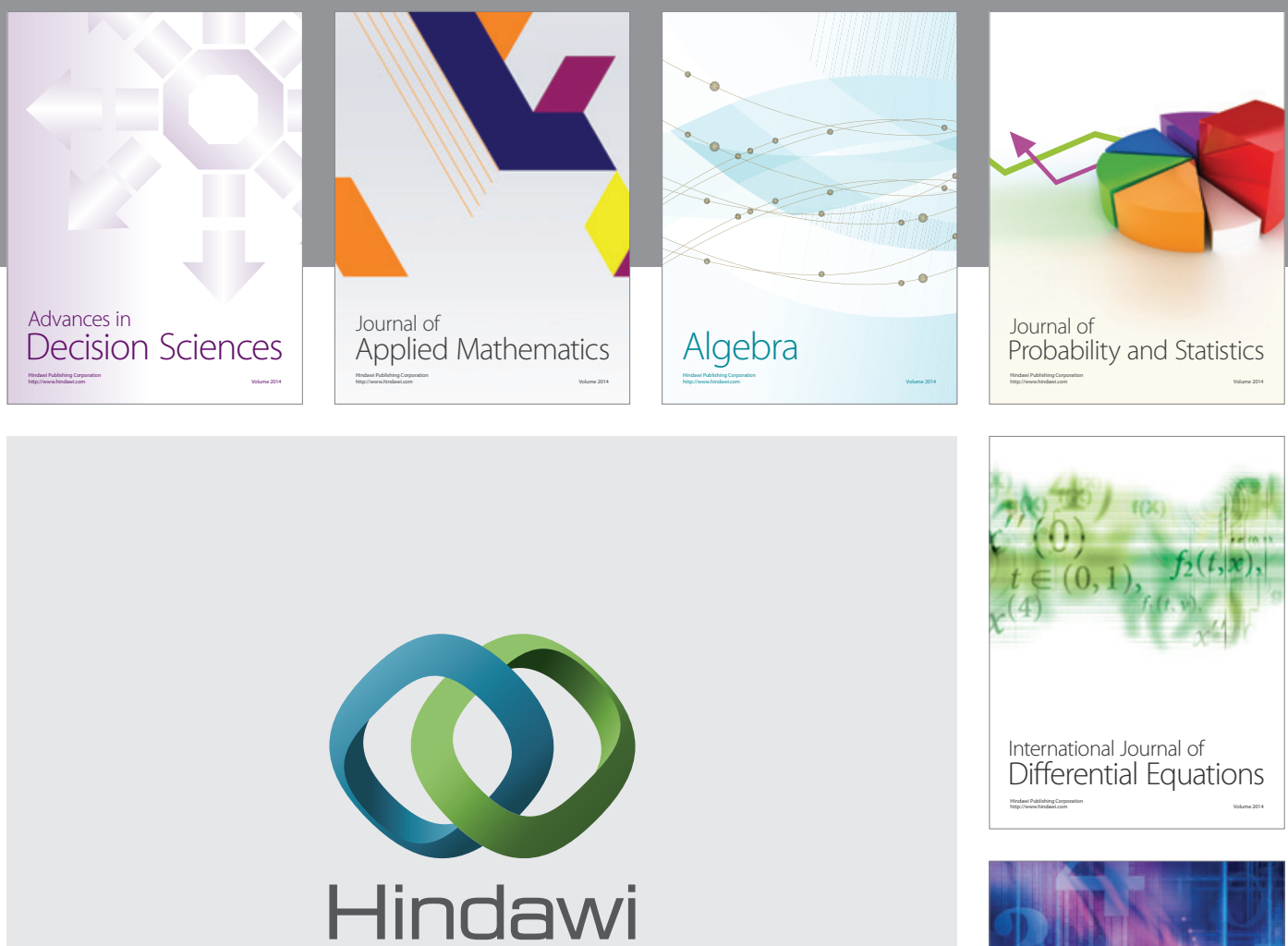

Submit your manuscripts at http://www.hindawi.com
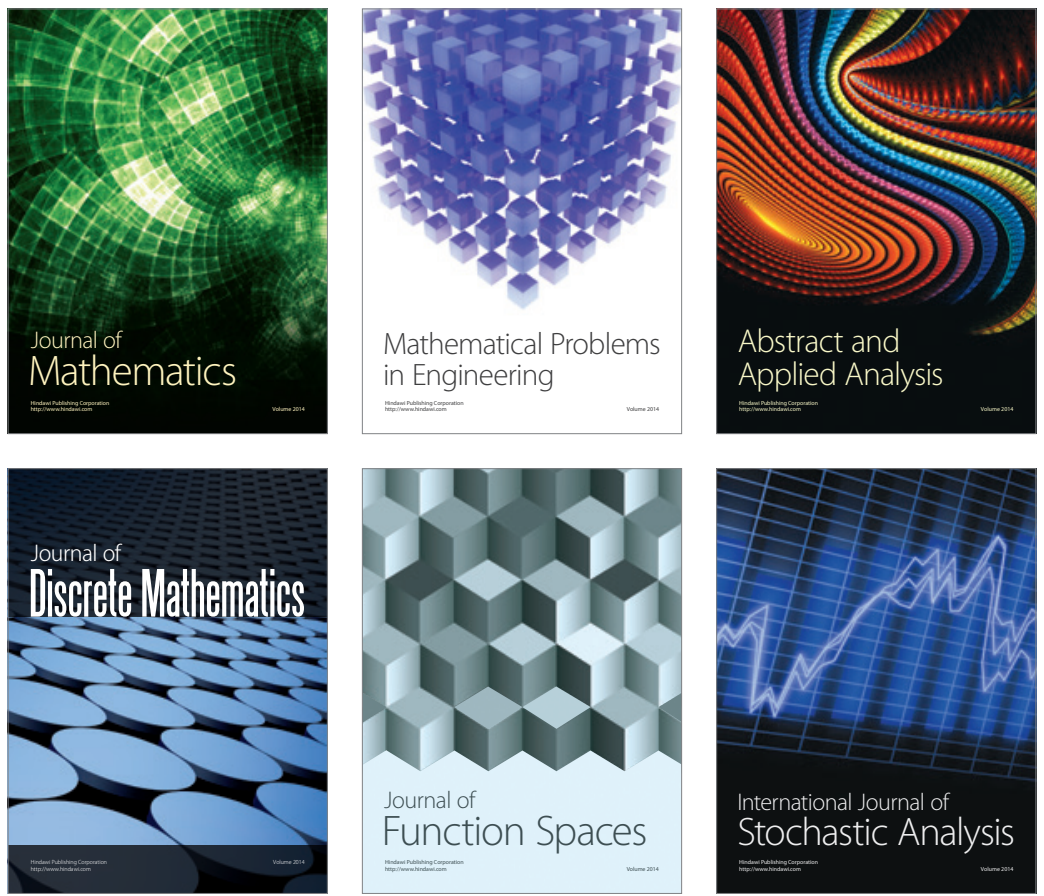

Journal of

Function Spaces

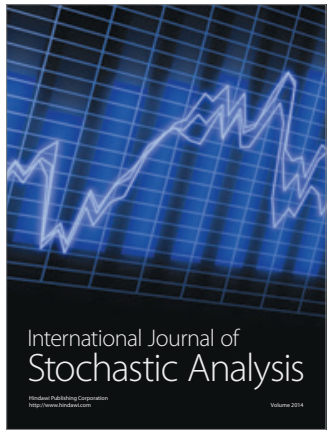

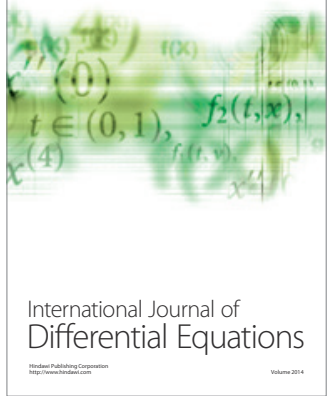
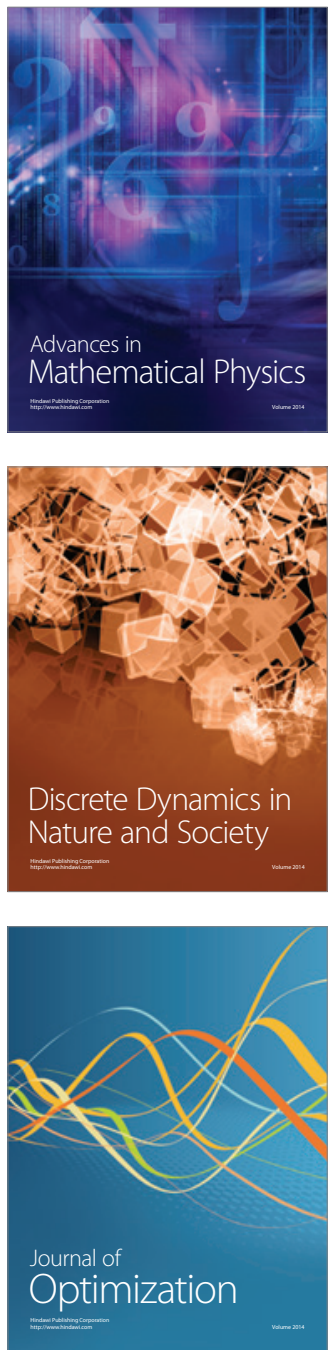